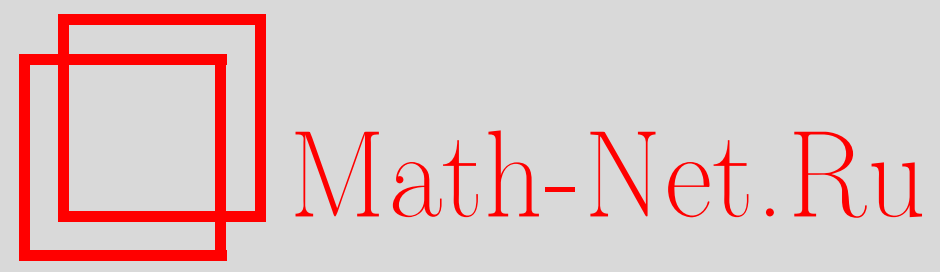

И. В. Павлов, О сохранении некоторых мартингальных неравенств для процессов более общего вида, Теория вероятн. $u$ ее примен., 1996, том 41, выпуск 2, 300-309

DOI: https://doi.org/10.4213/tvp2935

Использование Общероссийского математического портала Math-Net.Ru подразумевает, что вы прочитали и согласны с пользовательским соглашением http://www . mathnet.ru/rus/agreement

Параметры загрузки:

IP : 54.224 .187 .69

26 апреля 2023 г., 14:22:05

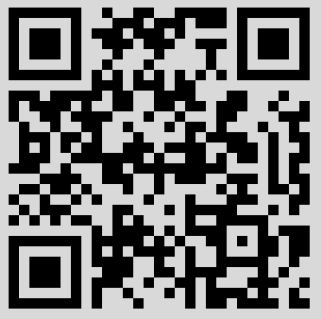


(C) 1996 г.

ПАВЛОВ И. В.*

\section{О СОХРАНЕНИИ НЕКОТОРЫХ МАРТИНГАЛЬНЫХ НЕРАВЕНСТВ ДЛЯ ПРОЩЕССОВ БОЛЕЕ ОБЩЕГО ВИДА}

В статье дается обобщение некоторых неравенств, справедливых для полумартингалов, на более широкий класс пропессов при дополнительных ограничениях на множество остановки.

Ключевые слова и фразы: мартингал, полумартингал, марковский момент, марковский момент первого достижения, множество остановки.

1. Введение. Предположим, что имеется случайная последовательность $g_{1}, g_{2}, \ldots, g_{n}, \ldots$, заданная на основном вероятностном пространстве $(\Omega, \mathcal{F}, \mathbf{P})$, которая является полумартингалом относительно неубывающей системы $\sigma$-подалгебр $\mathcal{F}_{n} \subset \mathcal{F}_{n+1} \subset \mathcal{F}, n=1,2, \ldots$. Пусть $\tau, \nu$ - некоторые марковские (относи тельно системы $\mathcal{F}_{n}, n=1,2, \ldots$ ) моменты такие, что $\mathbf{P}\{\tau \leqslant \nu\}=1$. Тогда при соответствуюших условиях ограниченности справедливы известные неравенства для полумартингалов вида

$$
\mathrm{E} g_{1} \leqslant \mathrm{E} g_{\tau} \leqslant \mathrm{E} g_{\nu}
$$

(где $\mathbf{E}$ - символ математического ожидания), если $g_{n}$ - субмартингал, и обратные неравенства, если $g_{n}$ - супермартингал (см. [1], [2] и пр.). Далее вводится более общий класс $S$-монотонных процессов (см. ниже п. 4), включающий в себя полумартингалы как частный случай, для которого справедливы ослабленные варианты указанных неравенств вида

$$
\begin{array}{lll}
\mathbf{E} g_{\tau} \geqslant \min _{n=1, \ldots, N} \mathbf{E} g_{n}, & \text { если } & \mathbf{P}\{\tau \leqslant N\}=1, \\
\mathbf{E} g_{\tau} \geqslant \inf _{n \geqslant 1} \mathbf{E} g_{n}, & \text { если } & \mathbf{P}\{\tau<\infty\}=1,
\end{array}
$$

при некоторых дополнительных условиях на марковский момент $\tau$. А именно, рассматриваются только марковские моменты «первого достижения» при некоторых ограничениях типа монотонности на траницу множества остановки (п. 4). Примером $S$-монотонного процесса, не являющегося полумартингалом, может служить, в частности, стандартное

*МГТУ им. Н. Э. Баумана, кафедра высшей математики, 2-я Бауманская, 5, 107005 Москва, Россия. 
выборочное среднее $g_{n}=\left(z_{1}+z_{2}+\cdots+z_{n}\right) / n, n=1,2 \ldots$ (где $z_{1}, \ldots$, $z_{n}, \ldots$ - последовательность независимых одинаково распределенных случайных величин). Указанные неравенства вида (1) позволяют получать полезные оценки и могут применяться в различных приложениях, таких как построение последовательных тестов для проверки гипотез типа рассмотренных в [3]-[6] и др., а также в связанных с ними задачах на построение последовательных доверительных границ и др. Более подробно соответствуюшие приложения предполагается рассмотреть в последующих публикациях.

2. Основные неравенства. Случай дискретного времени. Пусть $x_{n}, n=1,2 \ldots$, - неоднородный марковский процесс с дискретным временем, принимаюший значения из измеримого фазового пространства $(X, \mathfrak{B}), \mathcal{F}_{n}=\sigma\left(x_{1}, \ldots, x_{n}\right)$ - неубывающая система $\sigma$-подалгебр, связанная с пропессом $x_{n}$, и $\pi(n, x, m, \Gamma), \Gamma \in \mathfrak{B}$, - переходная функция процесса, удовлетворяющая обычным условиям регулярности (см., например, [2, с. 595]).

Далее будем рассматривать только марковские (относительно неубывающей системы $\left.\mathcal{F}_{n}, n=1,2, \ldots\right)$ моменты «первого достижения», т.е. марковские моменты (сокращенно м.м.) вкда

$$
\tau=\min \left\{n: x_{n} \in D_{n}\right\}
$$

где $D_{n} \in \mathfrak{B}, n=1,2 \ldots$, - та или иная заданная система множеств остановки. Если событие $x_{n} \in D_{n}$ не наступает ни при каком конечном $n$, то в (2) полагаем $\tau=\infty$. Класс марковских моментов первого достижения (м.м.п.д.) будем обозначать $\mathfrak{M}_{0}$, в отличие от класса всех марковских моментов $\mathfrak{M}$.

Пусть для каждого $n \geqslant 1$ на множестве состояний $X$ прощесса задана некоторая измеримая функция $g_{n}(x)$. Далее будем использовать сокращенные обозначения для случайных величин: $g_{n}\left(x_{n}\right)=g_{n}\left[x_{n}(\omega)\right]$, $g_{\tau}\left(x_{\tau}\right)=g_{\tau(\omega)}\left[x_{\tau(\omega)}(\omega)\right]$, или, еше короче, $g_{n}=g_{n}\left(x_{n}\right), g_{\tau}=g_{\tau}\left(x_{\tau}\right)$ и т.п. Аналогично для интегралов вида $\mathbf{E} g_{n}=\int_{\Omega} g_{n}\left[x_{n}(\omega)\right] \mathbf{P}(d \omega)$ будем использовать сокращенную запись $\int g_{n} d \mathbf{P}$ и т.п.

Предположим, что для каждого фиксированного момента времени $n$ математическое ожидание случайной величины (с.в.) $g_{n}$ удовлетворяет неравенству вида $\mathbf{E} g_{n} \geqslant(\leqslant) C, n=1,2, \ldots$, где константа $C$ не зависит от $n$. Рассмотрим вопрос о том, для каких м.м. $\tau \in \mathfrak{M}_{0}$ можно утверждать, что $\mathbf{E} g_{\tau} \geqslant(\leqslant) C$.

Всюду далее будем предполагать, что

$$
\mathbf{E}\left|g_{n}\right|<\infty, \quad n=1,2, \ldots .
$$

Пусть $m>n \geqslant 1$. Рассмотрим условное математическое ожидание $\mathbf{E}\left(g_{m} \mid \mathcal{F}_{n}\right)$ (сушествуюшее и Р-п.в. конечное в силу (3)), для которого имеет место равенство $\mathrm{E}\left(g_{m} \mid \mathcal{F}_{n}\right)=\varphi_{n m}\left(x_{n}\right),(\mathbf{P}$-п.в. $)$, где $\varphi_{n m}(x)=$ 
$\int_{X} g_{m}(y) \pi(n, x, m, d y)$ - величина, имеюшая смысл «прогноза» на момент $m$ среднего значения с.в. $g_{m}$, если в момент $n$ процесс находится в состоянии $x$.

Введем множества на пространстве состояний $X$

$$
G_{n m}^{+}=\left\{x: \varphi_{n m}(x) \geqslant g_{n}(x)\right\}, \quad G_{n m}^{-}=\left\{x: \varphi_{n m}(x) \leqslant g_{n}(x)\right\},
$$

имеющие следующий смысл. Если $x_{n} \in G_{n m}^{+}$в момент времени $n$, то прогнозируемое на момент $m>n$ среднее значение процесса $g$ не меньше, чем уже имеюшееся в данный момент. Если $x_{n} \in G_{n m}^{-}$, то прогнозируемое значение не больше имеющегося. Обозначим также $G_{n}^{-}=\cap_{m>n} G_{n m}^{-}$, $G_{n}^{+}=\cap_{m>n} G_{n m}^{+}-$области соответственно отрицательного и положительного прогноза из момента $n$ на все будушие моменты.

Пусть $D_{n}, n=1,2, \ldots$, - последовательность множеств остановки, определяющая м.м.п.д. в (2).

Марковский момент $\tau \in \mathfrak{M}_{0}$ назовем слабо положительным для процесса $g_{n}$, если для любой пары $n<m$ выполняется (с точностью до множеств нулевой меры) одно из двух соотношений включения: либо $D_{n} \subset G_{n m}^{-}$, либо $\bar{D}_{n} \subset G_{n m}^{+}$, где $\bar{D}_{n}=X \backslash D_{n}$ - область продолжения наблюдений для момента $n$. Класс м.м. $\tau$, слабо положительных для процесса $g_{n}$, будем обозначать $K_{g}^{+}$. Аналогично, м.м. $\tau \in \mathfrak{M}_{0}$ назовем положительным для $g_{n}$, если $\bar{D}_{n} \subset G_{n}^{+}$для любого $n=1,2, \ldots$ Класс таких м.м. обозначим $\bar{K}_{g .}^{+}, \bar{K}_{g}^{+} \subset K_{g}^{+}$. Ясно, что если $g_{n}-$ субмартингал, то $\bar{K}_{g}^{+}=K_{g}^{+}=\mathfrak{M}_{0}$.

Предположим, что $\tau \in{\overline{K_{g}}}_{g}$ и $\mathbf{E} g_{\tau}$ существует. Из определения класса $\bar{K}_{g}^{+}$достаточно очевидно, что в этом случае должно быть, например, (при соответствуюших условиях ограниченности)

$$
\mathbf{E} g_{\tau} \geqslant \mathbf{E} g_{1}
$$

и, более того, если $\left(\tau_{1}, \tau_{2}\right) \in \bar{K}_{g}^{+}$и $\tau_{1} \leqslant \tau_{2}$ (Р-п.в.), то $\mathbf{E} g_{\tau_{1}} \leqslant \mathbf{E} g_{\tau_{2}}$ (см. ниже теорему 4). Однако класс $\bar{K}_{g}^{+}-$слишком узкий. Более интересным в этом смысле является класс $K_{g}^{+}$, для которого имеет место ослабленный вариант неравенств типа (4). Рассмотрим сначала случай, когда м.м. $\tau$ ограничен сверху константой $N<\infty$.

Теорема 1. Ecлu $\tau \in \bar{K}_{g}^{+} u \mathbf{P}\{\tau \leqslant N\}=1$, mo

$$
\mathbf{E} g_{\tau} \geqslant \min _{n=1, \ldots, N} \mathbf{E} g_{n} \text {. }
$$

Д о к а 3 а т е л ь с т в о. В силу (3) $\mathbf{E}\left|g_{\tau}\right| \leqslant \operatorname{Emax}_{n=1, \ldots, N}\left|g_{n}\right|<\infty$. Имеем $\mathrm{E} g_{\tau}=\int g_{\tau} d \mathrm{P}=\sum_{n=1}^{N} \int_{A_{n}} g_{n} d \mathrm{P}$, где $A_{n}=\{\omega: \tau(\omega)=n\}$. Так как $A_{N}=\{\tau=N\}=\{\tau>N-1\} \in \mathcal{F}_{N-1}$, то

$$
\int_{A_{N}} g_{N} d \mathbf{P}=\int_{A_{N}} \mathbf{E}\left(g_{N} \mid \mathcal{F}_{N-1}\right) d \mathbf{P}=\int_{A_{N}} \varphi_{N-1, N}\left(x_{N-1}\right) d \mathbf{P} .
$$


Таким образом,

$$
\mathbf{E} g_{\tau}=\sum_{n=1}^{N-2} \int_{A_{n}} g_{n} d \mathbf{P}+\int_{A_{N-1}} g_{N-1} d \mathbf{P}+\int_{A_{N}} \varphi_{N-1, N}\left(x_{N-1}\right) d \mathbf{P} .
$$

Поскольку $\tau \in K_{g}^{+}$, то выполняется одно из двух условий. Либо $\varphi_{N-1, N}(x) \leqslant g_{N-1}(x)$ при всех $x \in D_{N-1}$, и в этом случае

$$
\varphi_{N-1, N}\left(x_{N-1}\right) \leqslant g_{N-1}\left(x_{N-1}\right)
$$

всюду на множестве $A_{N-1}$. Либо $\varphi_{N-1, N}(x) \geqslant g_{N-1}(x)$ при всех $x \in \bar{D}_{N-1}$, и в этом случае

$$
\varphi_{N-1, N}\left(x_{N-1}\right) \geqslant g_{N-1}\left(x_{N-1}\right)
$$

всюду на множестве $A_{N}$. Введем новый м.м. $\tau_{1}$ с последовательностью множеств остановки $D_{n}^{1}$, определяемой следующим образом. При $n<$ $N-1$ полагаем по-прежнему $D_{n}^{1}=D_{n}$. При $n=N-1$ положим $D_{n-1}^{1}=\varnothing$, если выполняется условие (8), и $D_{n-1}^{1}=X$, если выполняется условие (9). В первом случае

$$
\begin{aligned}
\mathbf{E} g_{\tau_{1}}= & \sum_{n=1}^{N-2} \int_{A_{n}} g_{n} d \mathbf{P}+\int_{A_{N-1}} \varphi_{N-1, N}\left(x_{N-1}\right) d \mathbf{P} \\
& +\int_{A_{N}} \varphi_{N-1, N}\left(x_{N-1}\right) d \mathbf{P} \leqslant \mathbf{E} g_{\tau}
\end{aligned}
$$

Во втором случае также

$$
\begin{aligned}
\mathbf{E} g_{\tau_{1}}= & \sum_{n=1}^{N-2} \int_{A_{n}} g_{n} d \mathbf{P}+\int_{A_{N-1}} g_{N-1}\left(x_{N-1}\right) d \mathbf{P} \\
& +\int_{A_{N}} g_{N-1}\left(x_{N-1}\right) d \mathbf{P} \leqslant \mathbf{E} g_{\tau} .
\end{aligned}
$$

Далее, производя те же операции, что и на первом шаге, получаем

$$
\mathbf{E} g_{\tau_{1}}=\sum_{n=1}^{N-3} \int_{A_{n}} g_{n} d \mathbf{P}+\int_{A_{N-2}} g_{N-2}\left(x_{N-2}\right) d \mathbf{P}+\int_{\tau>N-2} h_{N-2}\left(x_{N-2}\right) d \mathbf{P}
$$

где $h_{N-2}(x)=\varphi_{N-2, N}(x)$, если на первом шаге выполнялось условие $(8)$, и $h_{N-2}(x)=\varphi_{N-2, N-1}(x)$, если на первом шаге выполнялось условие (9). Из того, что $\tau \in K_{g}^{+}$, далее снова вытекает, что $h_{N-2}\left(x_{N-2}\right) \leqslant$ $g_{N-2}\left(x_{N-2}\right)$ всюду на множестве $A_{N-2}$, либо $h_{N-2}\left(x_{N-2}\right) \geqslant g_{N-2}\left(x_{N-2}\right)$ всюду на множестве $\{\tau>N-2\}$. Так же, как на первом шаге, заменяя в первом случае множество остановки $D_{N-2}$ на пустое множество $\phi$, а во втором случае на все пространство $X$ и вводя соответствуюший этой замене новый м.м. $\tau_{2}$, получаем $\mathbf{E} g_{\tau_{2}} \leqslant \mathbf{E} g_{\tau_{1}} \leqslant \mathbf{E} g_{\tau}$ и т.д. На последнем $(N-1)$-м шаге этой процедуры имеем $\mathbf{E} g_{\tau_{N-1}} \leqslant \ldots \leqslant \mathbf{E} g_{\tau_{1}} \leqslant \mathbf{E} g_{\tau}$, где 
последний м.м. $\tau_{N-1}$ является по построению некоторым фиксированным моментом: $\tau_{N-1} \equiv i$, где $1 \leqslant i \leqslant N$. Таким образом, $\mathbf{E} g_{\tau} \geqslant \mathbf{E} g_{i} \geqslant$ $\min _{n=1, \ldots, N} \mathbf{E} g_{n}$.

Обобщение неравенства (5) на случай не обязательно ограниченного, но конечного с вероятностью 1 м.м. может быть получено усечением м.м. константой $N$ и переходом к пределу при $N \rightarrow \infty$ при наличии дополнительных условий ограниченности, обеспечивающих возможность предельного перехода под знаком интеграла. Обозначим $g_{n}^{+}=\max \left(0, g_{n}\right)$, $g_{n}^{-}=-\min \left(0, g_{n}\right)$.

Tеорема 2. Ecлu 1) $\left.\left.\tau \in K_{g}^{+}, 2\right) \mathbf{P}\{\tau<\infty\}=1,3\right) \mathbf{E s u p}_{n} g_{n}^{+}<\infty$, mo

$$
\mathbf{E} g_{\tau} \geqslant \inf _{n \geqslant 1} \mathbf{E} g_{n}
$$

I о к а з а т е л ь с т в о. В силу условия 3) математическое ожидание $\mathbf{E} g_{\tau}$ существует. Введем усеченный м.м. $\tau_{N}=\min (\tau, N)$. Поскольку $\mathbf{P}\{\tau<\infty\}=1$, то $g_{\tau_{N}} \rightarrow g_{\tau}$ (Р-п.в.) при $N \rightarrow \infty$. Для каждого конечного $N \tau_{N} \in K_{g}^{+}$, и, следовательно, $\mathbf{E} g_{\tau_{N}} \geqslant \min _{n=1, \ldots, N} \mathbf{E} g_{n} \geqslant \inf _{n \geqslant 1} \mathbf{E} g_{n}$, откуда, используя лемму Фату-Лебега и условие ограниченности 3), получаем: $\mathbf{E} g_{\tau}=\mathbf{E} \varlimsup \overline{\lim } g_{\tau_{N}} \geqslant \varlimsup \overline{\lim } \mathbf{E} g_{\tau_{N}} \geqslant \inf _{n \geqslant 1} \mathbf{E} g_{n}$ при $N \rightarrow \infty$.

Условие ограниченности сверху 3) не всегда удобно. В некоторых случаях процесс $g_{n}$ заведомо ограничен снизу, например, неотрицателен. В этих случаях вместо условия 3) могут использоваться другие условия. Обозначим $\Delta_{N}=\int_{\tau>N} g_{N} d \mathbf{P}=\mathbf{E}\left(g_{N}, \tau>N\right)$.

Теорема 3. Ecлu 1) $\tau \in K_{g}^{+}$, 2) $\mathbf{P}\{\tau<\infty\}=1$, 3) $g_{n} \geqslant 0, n=$ $1,2, \ldots$, 4) $\lim \Delta_{N}=0$, mo

$$
\mathbf{E} g_{\tau} \geqslant \inf _{n \geqslant 1} \mathbf{E} g_{n}
$$

Доказате ль с т в о. Для каждого конечного $N$, обозначая $\tau_{N}=\min (\tau, N)$, имеем

$$
\begin{aligned}
\mathbf{E} g_{\tau_{N}} & =\int_{\tau \leqslant N} g_{\tau_{N}} d \mathbf{P}+\int_{\tau>N} g_{\tau_{N}} d \mathbf{P}=\int_{\tau \leqslant N} g_{\tau} d \mathbf{P}+\Delta_{N} \\
& \geqslant \min _{n=1, \ldots, N} \mathbf{E} g_{n} \geqslant \inf _{n \geqslant 1} \mathbf{E} g_{n} .
\end{aligned}
$$

В силу условий 2$), 3)$ имеем: $\lim \int_{\tau \leqslant N} g_{\tau} d \mathbf{P}=\mathbf{E} g_{\tau}$ при $N \rightarrow \infty$, откуда после предельного перехода в (12) следует (11).

Условие $\lim \Delta_{N}=0$ выполняется, например, если $\mathbf{P}\{\tau<\infty\}=1$ и пропесс $g_{n}\left(x_{n}\right)$ ограничен не обязательно при всех $x \in X$, а лишь в области продолжения наблюдений: $g_{n}(x) \leqslant c<\infty, x \in \bar{D}_{n}, n=1,2, \ldots$, так как в этом случае $\Delta_{N} \leqslant c \mathbf{P}\{\tau>N\} \longrightarrow 0$.

Для класса м.м. $\bar{K}_{g}^{+}$могут быть аналогичным образом установлены более сильные неравенства. Например, имеет место следуюшая приводимая без доказательства 
Теорема 4. Ecлu 1) $\tau_{1} \in \bar{K}_{g}^{+}, \tau_{2} \in \bar{K}_{g}^{+}$, 2) $\mathbf{P}\left\{\tau_{1} \leqslant \tau_{2}<\infty\right\}=1$, 3) $\operatorname{Esup}_{n} g_{n}^{+}<\infty$, mo

$$
\mathbf{E} g_{\tau_{2}} \geqslant \mathbf{E} g_{\tau_{1}}
$$

Теорема 4 справедлива и для несколько более широкого класса м.м. первого достижения таких, что $\bar{D}_{n} \subset G_{n, n+1}^{+}, n=1,2, \ldots$.

Приведенные результаты очевидным образом переформулируются на случай обратных неравенств. Марковский момент первого достижения $\tau$ назовем слабо отрицательным для процесса $g_{n}$, если для любой пары $m>n$ выполняется одно из двух соотношений включения: либо $D_{n} \subset G_{n m}^{+}$, либо $\bar{D}_{n} \subset G_{n m}^{-}$. Класс таких м.м. обозначим $K_{g}^{-}$. Марковский момент первого достижения $\tau$ назовем отрицательным для $g_{n}$, если $\bar{D}_{n} \subset G_{n}^{-}, n=1,2, \ldots$. Соответствуюший класс обозначим $\bar{K}_{g}^{-}$. Для указанных м.м. теоремы 1-4 остаются справедливыми в прежних формулировках с точностью до замены знаков + на -, inf на sup и неравенств (5), (10), (11), (13) на обратные.

Если процесс $g_{n}=g_{n}\left(x_{n}\right)$ является субмартингалом относительно семейства $\mathcal{F}_{n}$, то $\bar{K}_{g}^{+}=K_{g}^{+}=\mathfrak{M}_{0}$, т.е. любой м.м. $\tau \in \mathfrak{M}_{0}$ является в этом случае положительным и слабо положительным для $g_{n}$. Если $g_{n}-$ мартингал, то $\bar{K}_{g}^{-}=K_{g}^{-}=\bar{K}_{g}^{+}=K_{g}^{+}=\mathfrak{M}_{0}$. Приведенные неравенства, тем самым, включают как частный случай аналогичные известные неравенства для полумартингалов и мартингалов (при дополнительном условии, что процесс $x_{n}-$ марковский и $\left.\tau \in \mathfrak{M}_{0}\right)$.

Основной интерес для рассматриваемых далее приложений представляют слабо положительные (отрицательные) марковские моменты.

3. Случай непрерывного времени. Пусть $\left(x_{t}, \mathcal{F}_{t}\right), t \in T=$ $(0, \infty)$, - марковский процесс с непрерывным временем, заданный на полном вероятностном пространстве $(\Omega, \mathcal{F}, \mathbf{P})$ и принимающий значения в фазовом пространстве $(X, \mathfrak{B})$, где $X$ - локально компактное сепарабельное метрическое пространство, $\mathfrak{B}$ есть $\sigma$-алгебра его борелевских подмножеств. Неубывающее семейство $\sigma$-алгебр $\mathcal{F}_{t}$ предполагается непрерывным справа, а траектории процесса $x_{t}$ - непрерывными справа с вероятностью 1. Указанные условия регулярности относятся к числу стандартных. Нереходную функцию процесса обозначим через $\pi(t, x, u, \Gamma), t \leqslant u, \Gamma \in \mathfrak{B}$.

Марковский момент первого достижения

$$
\tau=\inf \left\{t>0:\left(t, x_{t}\right) \in D\right\}
$$

задается множеством остановки $D \subset X \times T$. В (14) полагается $\tau=\infty$, если событие $\left(t, x_{t}\right) \in D$ не наступает ни при каком конечном $t$. Далее будем предполагать, что множество остановки $D$ - замкнутое или открытое. Это условие является достаточным для того, чтобы определенный в (14) случайный момент был марковским (см. [1, гл. 1]). Через

3 Теория вероятностей и ее применения, № 2 
$D_{t}=\{x:(t, x) \in D\}, \bar{D}_{t}=\{x:(t, x) \in \bar{D}\}$ будем обозначать сечения множества остановки $D$ и множества продолжения наблюдений $\bar{D}=X \times T \backslash D$ при фиксированном $t$.

Пусть $g_{t}(x)$ - заданная на $X$ для каждого $t$ измеримая функция и $g_{t}=g_{t}\left(x_{t}\right)$ - соответствующий одномерный случайный процесс. Траектории процесса $g_{t}$ предполагаются непрерывными справа по $t$ c вероятностью 1. Так же, как выше, введем при $t<u$ функцию

$$
\varphi_{t u}(x)=\int_{X} g_{u}(y) \pi(t, x, u, d y)
$$

и множества $G_{t u}^{+}=\left\{x: \varphi_{t u}(x) \geqslant g_{t}(x)\right\}, G_{t u}^{-}=\left\{x: \varphi_{t u}(x) \leqslant g_{t}(x)\right\}$, имеющие прежний смысл.

Марковский момент первого достижения $\tau$ назовем слабо положительным для процесса $g_{t}$, если для любой пары $t<u$ выполняется одно из двух соотношений включения: либо $D_{t} \subset G_{t u}^{-}$, либо $\bar{D}_{t} \subset G_{t u}^{+}$. Класс таких м.м. по-прежнему обозначим $K_{g}^{+}$. Приведенные выше для случая дискретного времени неравенства достаточно просто переносятся на непрерывный случай при следующем дополнительном условии. Пусть $A_{\delta}$ - событие, состоящее в том, что $\left(t, x_{t}\right) \in D$ при всех $\tau \leqslant t<\tau+\delta$ и пусть $A=\cup_{\delta>0} A_{\delta}=\lim _{\delta \rightarrow 0} \uparrow A_{\delta}$. Марковский момент первого достижения $\tau$ такой, что $\mathbf{P}\{\tau<\infty\}=1$, назовем $\delta$-регулярным, если $\mathbf{P}(A)=1$, т.е. с вероятностью 1 процесс после попадания в множество остановки остается в нем еще некоторое строго положительное время, или, другими словами, вероятность «касания» множества остановки, не заходя в него, равна нулю. Проверка этого условия во многих случаях является очевидной. Например, оно заведомо выполняется, если множество остановки - поглощающее или открытое. Далее, имеет место, например, следугший аналог теоремы 3 для случая непрерывного времени.

Теорема 5. Если 1) $\delta$-регулярный м.м. $\tau \in K_{g}^{+}$, 2) $\mathbf{P}\{\tau<\infty\}=1$, 3) $\operatorname{Esup}_{t>0} g_{t}^{+}<\infty, m o$

$$
\mathbf{E} g_{\tau} \geqslant \inf _{t>0} \mathbf{E} g_{t}
$$

Д ок аз а т е ль с т в о. Рассмотрим разбиение временно́й оси дискретными моментами времени $S_{n}=\left\{t_{1}^{n}, t_{2}^{n}, \ldots\right\}$, где $t_{k}^{n}=k / 2^{n}, k=$ $1,2, \ldots, n=1,2, \ldots$, и соответствуюший этому разбиению вложенный марковский процесс с дискретным временем (т.е. исходный процесс, рассматриваемый в моменты $\left.t_{k}^{n}\right)$. Пусть $\tau_{n}=\min \left\{t: t \in S_{n}, x_{t} \in D_{t}\right\}-$ coответствующий $n$-му разбиению м.м. В используемых предположениях $\tau_{n} \downarrow \tau, x_{\tau_{n}} \rightarrow x_{\tau}, g_{\tau_{n}} \rightarrow g_{\tau}$ (P-п.в.) при $n \rightarrow \infty$. Для каждого разбиения по теореме 2 имеем $\mathbf{E} g_{\tau_{n}} \geqslant \inf _{t \in S_{n}} \mathbf{E} g_{t} \geqslant \inf _{t>0} \mathbf{E} g_{t}$, откуда после предельного перехода при $n \rightarrow \infty$, используя условие 3) и лемму Фату-Лебега, получаем $\mathbf{E} g_{\tau} \geqslant \varlimsup \mathbf{\operatorname { l i m }} \mathbf{E} g_{\tau_{n}} \geqslant \inf _{t>0} \mathbf{E} g_{t}$.

Условие ограниченности 3) выполняется, если, например, процесс $g_{t}$ ограничен сверху: $g_{t} \leqslant c<\infty$ при всех $t>0$. Вместо него в теоре$\max 2,4,5$ может использоваться условие равномерной интегрируемости 
семейства $g_{t}, t \in T$, и некоторые другие варианты условий ограниченности.

4. $S$-монотонные процессы. Пусть $\left(g_{t}, \mathcal{F}_{t}, \mathbf{P}\right), t \in T$, - одномерный случайный пропесс, $g_{t} \in \mathbf{R}_{1}=(-\infty, \infty)$, где $T=(0, \infty)$, либо $T=1,2, \ldots$. Через $\operatorname{sgn} z=\left\{\begin{array}{cc}1, & z \geqslant 0, \\ -1, & z \leqslant 0,\end{array}\right.$ обозначим знак величины $z \in \mathbf{R}_{1}$, предполагая, что sgn 0 определяется неоднозначно и может принимать значения -1 и 1 .

Процесс $g_{t}, t \in T$, назовем $S$-монотонным, если для любой пары $t<u,(t, u) \in T$, знак разности $g_{t}-\mathbf{E}\left(g_{u} \mid \mathcal{F}_{t}\right)$ зависит только от значения $g_{t}$ и функция

$$
S\left(g_{t}\right)=\operatorname{sgn}\left[g_{t}-\mathbf{E}\left(g_{u} \mid \mathcal{F}_{t}\right)\right]
$$

монотонна по $g_{t}$ (Р-п.в.). Или, более точно, если существует (с учетом неоднозначности величины sgn 0) такой вариант определения функции (16), что она монотонно не убывает или монотонно не возрастает по $g_{t}$. Прощесс $g_{t}, t \in T$, назовем $S$-возрастающим ( $S$-убывающим), если функция (16) монотонно не убывает (не возрастает) по $g_{t}$.

Частным случаем $S$-монотонных процессов, очевидно, являются мартингалы и полумартингалы. Действительно, нетрудно видеть, что для субмартингала $S\left(g_{t}\right) \equiv-1$, а для супермартингала $S\left(g_{t}\right) \equiv 1$. Таким образом, любой полумартингал одновременно является $S$-возрастающим и $S$-убываюшим процессом.

3 а м е ч а н и е. При однозначном определении величины $\operatorname{sgn} 0$, например, если положить $\operatorname{sgn} 0=-1$, субмартингал остается, а супермартингал может не быть $S$-монотонным процессом, так как для субмартингала при этом $S\left(g_{t}\right) \equiv-1$, а для супермартингала не обязательно $S\left(g_{t}\right) \equiv 1$. Использование в определении неоднозначности величины sgn 0 связано с этим чисто формальным затруднением.

Примером $S$-монотонного процесса, не являющегося полумартингалом, может служить процесс $g_{n}=\left(z_{1}+\cdots+z_{n}\right) / n, n=1,2, \ldots$, где $z_{1}, \ldots, z_{n}, \ldots$ - независимые одинаково распределенные с.в. с конечным средним $\mathbf{E} z_{1}=a$. В этом случае $g_{n}-\mathbf{E}\left(g_{m} \mid \mathcal{F}_{n}\right)=((m-n) / m)\left(g_{n}-a\right)$, $n<m$, тем самым, $S\left(g_{n}\right)=-1$ при $g_{n}<a$ и $S\left(g_{n}\right)=1$ при $g_{n} \geqslant a$, и процесс $g_{n}-S$-возрастающий.

Пусть так же, как и выше, имеется одномерный случайный процесс $g_{t}=g_{t}\left(x_{t}\right)$, заданный на марковском случайном пропессе $\left(x_{t}, \mathcal{F}_{t}, \mathbf{P}\right)$. Предположим, что м.м. $\tau$ задается множеством остановки, имеющим вид $D^{\prime}=\left\{(x, t): g_{t}(x) \geqslant b_{t}\right\}$, где $b_{t} \in[-\infty, \infty]$ - граница множества остановки. Далее будем использовать сокращенные обозначения

$$
D^{\prime}=\left\{g_{t} \geqslant b_{t}\right\}
$$

В соответствии с приведенными определениями м.м. $\tau$ со множеством остановки вида (17) является слабо положительным (слабо отрицатель- 
ным) для $g_{t}$, если процесс $g_{t}-S$-возрастающий ( $S$-убывающий). Аналогично, м.м. $\tau$, заданный множеством остановки вида

$$
D^{\prime \prime}=\left\{g_{t} \leqslant b_{t}\right\}
$$

является слабо отрицательным (слабо положительным) для $g_{t}$, если процесс $g_{t}-S$-возрастающий ( $S$-убывающий). При этом несущественно, строгие или нестрогие неравенства используются в (17), (18).

В соответствии с пп. 2, 3 для $S$-монотонных процессов и марковских моментов со множествами остановки вида $D^{\prime}, D^{\prime \prime}$ имеют место ослабленные варианты известных неравенств для полумартингалов. Так, для $S$-возрастаюшего процесса и м.м., заданного множеством остановки вида $D^{\prime}$, при соответствуюших условиях регулярности и ограниченности

$$
\mathbf{E} g_{\tau} \geqslant \inf _{t \in T} \mathbf{E} g_{t}
$$

Для $S$-убывающего процесса

$$
\mathbf{E} g_{\tau} \leqslant \sup _{t \in T} \mathbf{E} g_{t}
$$

Для м.м. со множеством остановки вида $D^{\prime \prime}$ в $(19),(20)$ имеют место обратные неравенства.

Отметим здесь обобщение одного известного неравенства для полумартингалов, сразу вытекающее из предыдущего. Пусть, например, $T$ - конечное множество: $T=1,2, \ldots, N, g_{t}$ - неотрицательный $S$ убывающий процесс, и множество остановки для м.м. $\tau$ имеет вид $D^{\prime}=$ $\left\{g_{t} \geqslant b\right\}$, где уровень $b>0$ не зависит от $t$. Полагая $\tau_{N}=\min (\tau, N)$, используя неравенство Чебышева и теорему 1 (точнее, ее аналог для слабо отрицательных м.м.), получаем

$$
\mathbf{P}\left\{\max _{t=1, \ldots, N} g_{t} \geqslant b\right\}=\mathbf{P}\left\{g_{\tau_{N}} \geqslant b\right\} \leqslant \frac{\mathbf{E} g_{\tau_{N}}}{b} \leqslant \frac{1}{b} \max _{t=1, \ldots, N} \mathbf{E} g_{t},
$$

что дает обобщение неравенства Дуба-Колмогорова для полумартингалов (см., например, [1], [2]) на случай $S$-убывающего процесса.

В некоторых случаях $S$-монотонный процесс может быть представлен в виде суммы субмартингала и супермартингала. Скажем, что $S$-возрастающий ( $S$-убывающий) процесс $g_{t}$ имеет центр $a_{t}$ в момент $t$, если существует такая не зависящая от $u$ константа $-\infty<a_{t}<\infty$, не обязательно единственная, что для любого $u>t, u \in T$ справедливо неравенство $g_{t} \leqslant(\geqslant) \mathbf{E}\left(g_{u} \mid \mathcal{F}_{t}\right)$ при $g_{t}<a_{t}$ и $g_{t} \geqslant(\leqslant) \mathbf{E}\left(g_{u} \mid \mathcal{F}_{t}\right)$ при $g_{t}>a_{t}$. $S$-монотонный процесс назовем процессом с одним центром, если для каждого $t$ существует центр $a_{t}$ такой, что $a_{t} \equiv a, t \in T$. Примером может служить указанный выше процесс $\widetilde{g}_{n}=\left(z_{1}+\cdots+z_{n}\right) / n-$ выборочное среднее.

Пусть $g_{t}$ есть $S$-убывающий пропесс с одним центром $a$. Нетрудно убедиться, что тогда каждый из процессов $\left(g_{t}-a\right)^{+},\left(g_{t}-a\right)^{-}$явля- 
ется субмартингалом. Из равенства $g_{t}=a+\left(g_{t}-a\right)^{+}-\left(g_{t}-a\right)^{-}$вытекает, тем самым, что $S$-убывающий процесс с одним центром может быть представлен в виде суммы субмартингала и супермартингала. Для $S$-возрастающего процесса такое представление, по-видимому, не всегда возможно. Заметим, что если $S$-возрастающие процессы, не являющиеся полумартингалами, встречаются довольно часто, то значительно труднее привести содержательный пример $S$-убывающего процесса, не являющтегося полумартингалом.

Наряду с данным выше определением $S$-монотонных процессов будем использовать еще одно. Пусть $z_{t}$ - еще один одномерный процесс, согласованный с семейством $\sigma$-алгебр $\mathcal{F}_{t}$. Процесс $g_{t}$ назовем $S$ возрастающим ( $S$-убываюшим) по $z_{t}$, если для любой пары $u>t,(u, t) \epsilon$ $T$, величина $\operatorname{sgn}\left[g_{t}-\mathbf{E}\left(g_{u} \mid \mathcal{F}_{t}\right)\right]$ зависит только от $z_{t}$ и существует вариант выбора функции $S\left(z_{t}\right)=\operatorname{sgn}\left[g_{t}-\mathbf{E}\left(g_{u} \mid \mathcal{F}_{t}\right)\right]$, такой, что она монотонно не убывает (не возрастает) по $z_{t}$.

Предположим, что одномерные процессы $g_{t}, z_{t}$ определены на марковском прогессе $\left(x_{t}, \mathcal{F}_{t}, \mathbf{P}\right)$, т.е. $g_{t}=g_{t}\left(x_{t}\right), z_{t}=z_{t}\left(x_{t}\right)$, и $g_{t}$ является $S$-возрастающим ( $S$-убывающим) по $z_{t}$. Неравенство (19) (неравенство (20)) остается при этом справедливым, если множество остановки имеет вид $D^{\prime}=\left\{z_{t} \geqslant b_{t}\right\}$. Если множество остановки имеет вид $D^{\prime \prime}=\left\{z_{t} \leqslant b_{t}\right\}$, то справедливы обратные неравенства.

\section{СПИСОК ЛИТЕРАТУРЫ}

1. Ширяев А. Н. Статистический последовательный анализ. М.: Наука, 1976, 271 с.

2. ЛІоэв M. Теория вероятностей. М.: ИЛ, 1962, 711 с.

3. Lorden G. 2-SPRT's and the modified Kiefer-Weiss problem of minimizing an expected sample size. - Ann. Statist., 1976, v. 4, № 2, p. 281-291.

4. Драгалин В. П., Новиков А. А. Асимптотическое решение задачи Кифера-Вайсса для процессов с независимыми приращениями. - Теория вероятн. и ее примен., 1987, т. XXXII, в. 4, с. 679-690.

5. Павлов И. В. Последовательная процедура различения многих сложных гипотез. - Теория вероятн. и ее примен., 1987, т. XXXII, в. 1, с. 149-153.

6. Павлов И. В. Последовательная процедура проверки сложных гипотез с примененйями к задаче Кифера-Вайсса. - Теория вероятн. и ее примен., 1990, т. XXXV, B. 2, c. 293-304. 